\title{
EFEITO DE HERBICIDAS UTILIZADOS EM CANA-DE- AÇÚCAR NO DESENVOLVIMENTO IN VITRO DO FUNGO ENTOMOPATOGÊNICO Metarhizium anisopliae (Metsch.) SOROKIN
}

\author{
EDUARDO A. D. DA COSTA * \\ MARCUS B. MATALLO * \\ JOSÉ E. M. ALMEIDA ** \\ ELISÂNGELA S. LOUREIRO *** \\ ALEXANDRE H. SANO ***
}

\begin{abstract}
Avaliou-se a compatibilidade dos herbicidas butafenacil, metribuzim, s-metolacloro, 2,4-D, glifosato, oxassulfurom e trifloxissulfuron no desenvolvimento do fungo entomopatogênico $M$. anisopliae (isolado IBCB 348), empregado para controle biológico de Mahanarva fimbriolata (Hemiptera: Cercopidae) conhecida como cigarrinhada-raiz da cana. Os herbicidas foram adicionados ao meio de cultura batata dextrose ágar (BDA) em temperatura aproximada de $45^{\circ} \mathrm{C}$ nas doses de campo mínimas e máximas recomendadas, fixandose o volume de calda em $200 \mathrm{~L} / \mathrm{ha}$. Após a solidificação do meio em placas de Petri, o fungo foi inoculado em três pontos eqüidistantes. As placas foram incubadas em câmara climatizada avaliandose o diâmetro médio das colônias formadas, 0 número de conídios por colônia e a viabilidade dos conídios do fungo após 20 e 48 horas da inoculação. Apenas o herbicida oxassulfurom, na menor dose testada (30 g/ha), foi classificado como tóxico ao $M$. anisopliae, sendo os demais classificados em ambas as doses como muito tóxicos. Quanto à viabilidade, somente os herbicidas oxassulfurom nas doses de 30 e $60 \mathrm{~g} / \mathrm{ha}$ não afetaram a germinação do fungo até 20 horas após a inoculação e o glifosato a 0,5 e $6,0 \mathrm{~L} / \mathrm{ha}$ até 48 horas. Os demais herbicidas mostraram-se incompatíveis, afetando o desenvolvimento e a germinação do $M$. anisopliae.
\end{abstract}

PALAVRAS-CHAVE: HERBICIDAS; CANA-DE-AÇÚCAR; Metarhizium anisopliae (Metsch.) SOROKIN.

* Pesquisador Científico, Doutor em Agronomia, Laboratório das Ciências das Plantas Daninhas, Instituto Biológico, Campinas, SP/Brasil (e-mail: aptauba@pratica.com.br/matallo@biologico.sp.gov.br).

** Pesquisador Científico, Doutor em Agronomia, Laboratório de Controle Biológico, Instituto Biológico, Campinas, SP/Brasil (e-mail: jmalmeida@biologico.sp.gov.br).

*** Engenheiro Agrônomo, Estagiário do Laboratório de Controle Biológico, Instituto Biológico, Campinas, SP/Brasil (e-mail: esloureiro@yahoo.com.br / ahsano@unesp.fcav.unesp.br). 


\section{INTRODUÇÃO}

A mecanização da colheita da cana-de-açúcar tem provocado alterações no comportamento da entomofauna desse agroecossistema. De acordo com MACEDO, CAMPOS e ARAÚJO (1997) tem sido observado aumento significativo na incidência de Mahanarva fimbriolata (Hemiptera: Cercopidae), a cigarrinha-da-raiz da cana-de-açúcar, com prejuízos de até $60 \%$ na produtividade dessas áreas.

O controle da M. fimbriolata por agentes biológicos, como os fungos entomopatogênicos, é uma das técnicas adotadas em programas de manejo integrado com o intuito de reduzir o uso de defensivos químicos e aumentar a proteção ao meio ambiente (ALVES et al., 1998). Dentre os agentes microbianos de controle, o fungo Metarhizium anisopliae vem sendo extensivamente empregado no controle biológico de $M$. fimbriolata pela alta eficiência e, principalmente, pelo baixo custo de produção e aplicação (COSTA et al., 2002).

A interação entre $M$. anisopliae com inseticidas e adjuvantes pode variar em função da natureza química do produto e sua concentração (BATISTA FILHO, ALMEIDA e LAMAS, 2001; COSTA et al., 2003), resultando em compatibilidade diferenciada de acordo com o produto. Nesse sentido, ALVES, MOINO JR. e ALMEIDA (1998) publicaram diversas tabelas de compatibilidade entre agrotóxicos e fungos entomopatogênicos importantes no controle microbiano de insetos com base em diversos autores. Afirmaram que a maioria das combinações com inseticidas exerce efeito aditivo. Apesar do grande número de agrotóxicos listados não há informações sobre a sua compatibilidade com herbicidas.

A interação entre microrganismos entomopatogênicos e herbicidas foi reportada por ÁVILA e MELHORANÇA (1999). Tais autores avaliaram a eficiência do vírus da poliedrose nuclear, utilizado no controle da lagarta da soja (Anticarsia gemmatalis), quando aplicado em mistura com herbicidas pósemergentes. Não foi constatada interferência desses produtos na eficácia do vírus.

O uso de herbicidas de diferentes grupos químicos na cultura da cana-de- açúcar constitui prática comum no manejo das plantas daninhas presentes no canavial, tanto em aplicações de préemergência como de pós-emergência. Seu emprego em combinação com entomopatógenos exige conhecimento da ação desses produtos sobre os microorganismos para determinar a sua compatibilidade e a viabilidade das estruturas de reprodução. Essa interação deve ser avaliada antes da recomendação de determinado agente químico, desempenhando importante papel em programas de manejo integrado de pragas (BATISTA FILHO, ALMEIDA e LAMAS, 2001).

O objetivo do presente trabalho foi verificar, in vitro, a ação tóxica dos herbicidas butafenacil, metribuzim, s-metolacloro, 2,4-D, glifosato, oxassulfurom e trifloxissulfurom sobre o fungo entomopatogênico $M$. anisopliae de ocorrência natural, ou artificialmente introduzido no agroecossistema da cana-de-açúcar.

\section{MATERIAL E MÉTODOS}

O trabalho foi desenvolvido no Laboratório de Controle Biológico do Instituto Biológico, Campinas (SP). Utilizou-se o isolado IBCB 348 de M. anisopliae originária desse Laboratório.

Estudou-se o efeito dos herbicidas listados na Tabela 1, nas doses mínima e máxima recomendadas pelo fabricante, avaliando-se o crescimento vegetativo e a conidiogênese dos fungos na presença e ausência desses produtos in vitro. A adição dos herbicidas ao meio batata dextrose ágar (BDA) foi realizada em concentrações equivalentes a $200 \mathrm{~L} /$ ha de calda.

Para avaliar os efeitos dos tratamentos no crescimento vegetativo e na produção de conídios do fungo, os herbicidas foram incorporados em erlenmeyers contendo $200 \mathrm{~mL}$ de meio de cultura batatadextrose ágar (BDA) a temperatura de $45^{\circ} \mathrm{C}$, após a autoclavagem. Com o meio ainda não solidificado, a mistura foi vertida em placas de Petri de $9 \mathrm{~cm}$ de diâmetro. Após a solidificação efetuou-se a inoculação do fungo na concentração de $1,0 \times 10^{8}$ conídios $/ \mathrm{mL}$, sendo confeccionadas 3 placas por tratamento. A inoculação foi realizada por meio de alça de platina em três pontos eqüidistantes entre si, totalizando 9 colônias do fungo. Dessas, 6 colônias foram aleatoriamente apontadas, resultando 
assim em 6 repetições por tratamento (ALVES et al., 1998).

\section{TABELA 1 - DESCRIÇÃO DOS TRATAMENTOS E DOSES DOS HERBICIDAS UTILIZADOS}

\begin{tabular}{|c|c|c|}
\hline \multirow[t]{2}{*}{ TRATAMENTOS } & \multicolumn{2}{|c|}{ DOSES UTILIZADAS (ha) } \\
\hline & M"NIMA & $M^{\prime} X I M A$ \\
\hline Testemunha & & \\
\hline butafenacil & $50,0 \mathrm{~m} \mathrm{~L}$ & $100,0 \mathrm{~mL}$ \\
\hline $2,4-D$ & $1,0 \mathrm{~L}$ & $2,0 \mathrm{~L}$ \\
\hline glifosato & $0,5 \mathrm{~L}$ & $6,0 \mathrm{~L}$ \\
\hline metribuzim & $0,5 \mathrm{~kg}$ & $0,7 \mathrm{~kg}$ \\
\hline oxassulfuron & $30,0 \mathrm{~g}$ & $60,0 \mathrm{~g}$ \\
\hline s-metolacloro & $2,0 \mathrm{~L}$ & $3,0 \mathrm{~L}$ \\
\hline trifloxissulfuron de $s$ dio & $5,0 \mathrm{~g}$ & $10,0 \mathrm{~g}$ \\
\hline
\end{tabular}

Depois da inoculação, as placas foram mantidas em câmara climatizada a $26 \pm 1^{\circ} \mathrm{C}$ e fotofase de 12 horas durante 12 dias. Utilizou-se régua para medir as colônias em dois sentidos ortogonais e determinar o diâmetro médio. As colônias foram retiradas das placas com bisturi e transferidas para tubos de ensaio, contendo $10 \mathrm{~mL}$ de água destilada estéril + espalhante adesivo Tween 80 . Realizaramse as diluições necessárias na suspensão original de cada tratamento e contagem do número de conídios em microscópio óptico, com o auxílio de câmara de Neubauer.

Os dados foram padronizados para o cálculo do fator de compatibilidade (Valor "T") proposto por ALVES, MOINO JR. e ALMEIDA (1998). Esse índice permite a classificação dos herbicidas em classes de compatibilidade conforme o efeito observado em relação aos parâmetros avaliados, sendo calculado pela fórmula:

$$
\mathrm{T}=\underline{20(\mathrm{VG})+80(\mathrm{SP})}
$$

\section{0}

na qual:

$T=$ valor corrigido do crescimento vegetativo e reprodutivo para classificação do produto;

$\mathrm{G}$ = porcentagem de crescimento vegetativo com relação à testemunha;

$\mathrm{SP}=$ porcentagem de esporulação (conidiogênese) com relação à testemunha.

Os valores calculados de "T" para a classificação do efeito dos herbicidas sobre o fungo foram comparados com os seguintes limites pré-estabelecidos: 0 a $30=$ muito tóxico; 31 a $45=$ tóxico; 46 a 60 = moderadamente tóxico; $>60$ = compatível.

Para avaliar a viabilidade do fungo foi confeccionada calda, contendo $100 \mathrm{~mL}$ de água destilada esterilizada adicionada de $0,02 \mathrm{~g}$ de fungo, além dos herbicidas nas concentrações máxima e mínima recomendadas (Tabela 1). Após 1 hora retirou-se alíquota de $0,1 \mathrm{~mL}$ de cada suspensão, espalhandoa com alça de Drigalsky em 4 placas de Petri contendo uma camada de meio de cultura BDA (consideradas como repetições). As placas foram mantidas em câmara climatizada por 20 e 48 horas a $26 \pm 1^{\circ} \mathrm{C}$ e fotofase de 12 horas. Com o auxílio de microscópio óptico, com aumento de 400 vezes, foi avaliada a germinação dos conídios em 4 campos por placa de Petri, quantificando-se a porcentagem de conídios germinados.

Todos os dados de crescimento vegetativo e produção de conídios foram transformados em log $x+10$ e os de porcentagem de germinação dos conídios em $\sqrt{x+0,5}$. Os dados foram submetidos à análise de variância pelo teste $F$, ao nível de $5 \%$ do erro experimental, e ao teste de Tukey $(P<0,05)$ para comparação entre as médias (BANZATTO e KRONKA, 1989). 


\section{RESULTADOS E DISCUSSÃO}

Apesar de interferirem na reprodução do fungo entomopatogênico $M$. anisopliae, reduzindo significativamente a produção de conídios (Tabela 2), os herbicidas glifosato na dose de 0,5 L/ha e oxassulfurom a 30 e $60 \mathrm{~g} / \mathrm{ha}$ não afetaram seu crescimento vegetativo. Essa diferença pode ser atribuída à variabilidade na suscetibilidade tanto do fungo como de seu isolado, conforme relataram ROBERTS e CAMPBELL (1977), citados por BATISTA FILHO, ALMEIDA e LAMAS (2001). Os demais tratamentos afetaram o crescimento vegetativo e reprodutivo do fungo, reduzindo o diâmetro das colônias e a produção de conídios estatisticamente. Butafenacil, 2,4-D, metribuzim e s-metolacloro foram os herbicidas que apresentaram maior redução no diâmetro das colônias (tanto nas doses mínimas como nas máximas), seguidos pelo trifloxissulfurom de sódio a 5,0 e 10,0 g/ha e glifosato a 6,0 L/ha. Observou-se que butafenacil, 2,4-D, glifosato, metribuzim e s-metolacloro ocasionaram a maior redução na produção de conídios, independente da dose, sendo semelhante à de trifloxissulfurom de sódio a 10,0 e $5,0 \mathrm{~g} / \mathrm{ha}$ e oxassulfurom a $60 \mathrm{e} 30 \mathrm{~g} / \mathrm{ha}$ (Tabela 2 ).

\section{TABELA 2 - DIÂMETRO MÉDIO DE COLÔNIAS (mm) E NÚMERO MÉDIO DE CONÍDIOS PRODUZIDOS POR COLÔNIA DE Metarhizium anisopliae NA PRESENÇA DE DIFERENTES CONCENTRAÇÕES DOS HERBICIDAS*}

\begin{tabular}{|c|c|c|}
\hline \multirow[b]{2}{*}{ Tratamentos } & \multicolumn{2}{|c|}{ Metarhizium anisopliae } \\
\hline & $\begin{array}{c}\text { Di metro de } \\
\text { col nias } \\
(\mathrm{m} \mathrm{m})\end{array}$ & $\begin{array}{c}\text { Produ } \\
\text { con dios }\left(10^{8} / \mathrm{m} L\right)\end{array}$ \\
\hline Testem unha & $1,78 \mathrm{a}$ & $2,58 \mathrm{a}$ \\
\hline butafenacil (50 m L/ha) & $0,71 \mathrm{c}$ & $0,71 \mathrm{~d}$ \\
\hline butafenacil (100 m L/ha) & $0,71 \mathrm{c}$ & $0,71 \mathrm{~d}$ \\
\hline $2,4-\mathrm{D}(1,0 \mathrm{~L} / \mathrm{ha})$ & $0,71 \mathrm{c}$ & $0,71 \mathrm{~d}$ \\
\hline $2,4-D(2,0 \mathrm{~L} / \mathrm{h} a)$ & $0,71 \mathrm{c}$ & $0,71 \mathrm{~d}$ \\
\hline glifos a to $(0,5 \mathrm{~L} / \mathrm{ha})$ & $1,79 a$ & $0,71 \mathrm{~d}$ \\
\hline glifos a to $(6,0 \mathrm{~L} / \mathrm{ha})$ & $1,10 \mathrm{~b}$ & $0,71 \mathrm{~d}$ \\
\hline m etribuzim $(0,5 \mathrm{~kg} / \mathrm{ha})$ & $0,71 \mathrm{c}$ & $0,71 \mathrm{~d}$ \\
\hline m etribuzim $(0,7 \mathrm{~kg} / \mathrm{ha})$ & $0,71 \mathrm{c}$ & $0,71 \mathrm{~d}$ \\
\hline oxassulfuron $(30 \mathrm{~g} / \mathrm{ha})$ & $1,85 \mathrm{a}$ & $1,46 b$ \\
\hline oxassulfuron $(60 \mathrm{~g} / \mathrm{ha})$ & $1,77 \mathrm{a}$ & $1,13 c$ \\
\hline $\mathrm{s}-\mathrm{m}$ etolacloro $(2,0 \mathrm{~L} / \mathrm{h} \mathrm{a})$ & $0,71 \mathrm{c}$ & $0,71 \mathrm{~d}$ \\
\hline $\mathrm{s}-\mathrm{m}$ etolacloro $(3,0 \mathrm{~L} / \mathrm{h}$ a $)$ & $0,71 \mathrm{c}$ & $0,71 \mathrm{~d}$ \\
\hline trifloxissulfuron de $\mathrm{s}$ dio $(5 \mathrm{~g} / \mathrm{ha})$ & $1,13 b$ & $1,11 \mathrm{c}$ \\
\hline trifloxissulfuron de $\mathrm{s}$ dio $(10 \mathrm{~g} / \mathrm{ha})$ & $1,11 \mathrm{~b}$ & $0,71 \mathrm{~d}$ \\
\hline \multicolumn{3}{|l|}{ Anßise de Vari ncia (resumo) } \\
\hline F (tratamentos) & $41,17^{* *}$ & $90,02 * *$ \\
\hline DM S & 0,37 & 1,08 \\
\hline $\mathrm{CV}(\%)$ & 16,86 & 28,5 \\
\hline
\end{tabular}

* dados transformados em log $x+10$. DMS = diferença mínima significativa.

** significativo ao nível de $5 \%$ de probabilidade. $\mathrm{CV}=$ coeficiente de variação.

Quando os dados foram submetidos à fórmula para determinação do valor T (Tabela 3 ) nenhum tratamento mostrou compatibilidade com o fungo $M$. anisopliae. Oxassulfurom a $30,0 \mathrm{~g} / \mathrm{ha}$ foi o único classificado como tóxico, sendo os demais herbicidas classificados como muito tóxicos (tanto nas doses máximas como nas mínimas). Esses resultados indicam a possibilidade da ocorrência de danos dessa natureza, pois sendo obtidos a partir de estudos in vitro com máxima exposição do fungo aos herbicidas podem mascarar a real interação com os fungos entomopatogênicos no solo (ALVES et. al., 1998). Processos de transformação e degradação regulam a biodisponibilidade dos herbicidas, tornando-os mais ou menos propensos a interargir no ambiente edáfico (MATALLO, 1999). 


\begin{tabular}{|c|c|c|}
\hline Tratamentos & $\mathrm{T}$ & Classifica ${ }^{a}$ o \\
\hline butafenacil $(50 \mathrm{~mL} / \mathrm{ha})$ & zero & Muito $t$ xico \\
\hline butafenacil $(100 \mathrm{~m} \mathrm{~L} / \mathrm{ha})$ & zero & Muito $t$ xico \\
\hline $2,4-D \quad(1,0 \mathrm{~L} / \mathrm{h}$ a $)$ & zero & Muito $t$ xico \\
\hline $2,4-D \quad(2,0 \mathrm{~L} / \mathrm{h}$ a $)$ & zero & Muito $t$ xico \\
\hline glifosato $(0,5 \mathrm{~L} / \mathrm{h}$ a $)$ & 20,22 & Muito $t$ xico \\
\hline glifosato $(6,0 \mathrm{~L} / \mathrm{ha})$ & 6,74 & Muito $t$ xico \\
\hline m etribuzim $(0,5 \mathrm{~kg} / \mathrm{ha})$ & zero & Muito $t$ xico \\
\hline m etribuzim $(0,7 \mathrm{~kg} / \mathrm{ha})$ & zero & Muito $t$ xico \\
\hline oxassulfuron $(30 \mathrm{~g} / \mathrm{ha})$ & 43,68 & $\mathrm{~T}$ xico \\
\hline oxassulfuron $(60 \mathrm{~g} / \mathrm{ha})$ & 29,91 & Muito $t$ xico \\
\hline $\mathrm{s}$-m etolacloro $(2,0 \mathrm{~L} / \mathrm{h}$ a $)$ & zero & Muito $t$ xico \\
\hline $\mathrm{s}-\mathrm{m}$ etolacloro $(3,0 \mathrm{~L} / \mathrm{h}$ a $)$ & zero & Muito $t$ xico \\
\hline trifloxissulfuron de $s$ dio $(5 \mathrm{~g} / \mathrm{ha})$ & 23,95 & Muito $t$ xico \\
\hline trifloxissulfuron de $s$ dio $(10 \mathrm{~g} / \mathrm{ha})$ & 13,33 & Muito $t$ xico \\
\hline
\end{tabular}

$\mathrm{T}=$ Fator de compatibilidade.

Quanto à viabilidade dos conídios do fungo (Tabela 4), os herbicidas butafenacil, 2,4-D, metribuzim e s-metolacloro foram os que mais afetaram a sobrevivência do $M$. anisopliae. Reduziram significativamente a porcentagem de germinação, tanto na leitura realizada 20 horas após incubação como na de 48 horas, independentemente da dose. Embora menores, reduções significativas na porcentagem de germinação dos conídios também foram verificadas para o trifloxissulfurom de sódio a 5 e $10 \mathrm{~g} / \mathrm{ha}$ em ambos períodos de leitura.

TABELA 4 - GERMINAÇÃO DE CONÍDIOS DO FUNGO Metarhizium anisopliae *

\begin{tabular}{|c|c|c|}
\hline \multirow[t]{2}{*}{ Tratam entos } & \multicolumn{2}{|c|}{ V ia bilid a de } \\
\hline & 20 horas & 48 horas \\
\hline testem unha & $9,92 \mathrm{a}^{1}$ & $10,03 \mathrm{a}$ \\
\hline butafenacil $(50 \mathrm{~m} \mathrm{~L} / \mathrm{ha})$ & $0,71 \mathrm{~d}$ & $0,71 \mathrm{~d}$ \\
\hline butafenacil $(100 \mathrm{~mL} / \mathrm{ha})$ & $0,71 \mathrm{~d}$ & $0,71 \mathrm{~d}$ \\
\hline $2,4-\mathrm{D} \quad(1,0 \mathrm{~L} / \mathrm{h}$ a $)$ & $0,71 \mathrm{~d}$ & $0,71 \mathrm{~d}$ \\
\hline $2,4-D \quad(2,0 \quad \mathrm{~L} / \mathrm{h}$ a $)$ & $0,71 \mathrm{~d}$ & $0,71 \mathrm{~d}$ \\
\hline glifos a to $(0,5 \mathrm{~L} / \mathrm{h}$ a $)$ & $6,15 \mathrm{~b}$ & 9,90 a \\
\hline glifos a to $(6,0 \mathrm{~L} / \mathrm{h}$ a $)$ & $6,59 \mathrm{~b}$ & $10,03 \mathrm{a}$ \\
\hline m e tribuzim $(0,5 \mathrm{~kg} / \mathrm{h} \mathrm{a})$ & $0,71 \mathrm{~d}$ & $0,71 \mathrm{~d}$ \\
\hline m e tribuzim $(0,7 \mathrm{~kg} / \mathrm{h} \mathrm{a})$ & $0,71 \mathrm{~d}$ & $0,71 \mathrm{~d}$ \\
\hline oxassulfuron $(30 \mathrm{~g} / \mathrm{h}$ a $)$ & 9,68 a & $10,03 \mathrm{a}$ \\
\hline oxassulfuron $(60 \mathrm{~g} / \mathrm{ha})$ & 9,64 a & 9,96 a \\
\hline $\mathrm{s}-\mathrm{m}$ e tola cloro $(2,0 \mathrm{~L} / \mathrm{h} \mathrm{a})$ & $0,71 \mathrm{~d}$ & $0,71 \mathrm{~d}$ \\
\hline $\mathrm{s}-\mathrm{m}$ e tola cloro $(3,0 \mathrm{~L} / \mathrm{h}$ a $)$ & $0,71 \mathrm{~d}$ & $0,71 \mathrm{~d}$ \\
\hline trifloxissulfuron de $s$ dio $(5 \mathrm{~g} / \mathrm{h}$ a) & $4,35 \mathrm{c}$ & $4,74 \mathrm{~b}$ \\
\hline trifloxis sulfuron de $s$ dio $(10 \mathrm{~g} / \mathrm{ha})$ & $1,59 \mathrm{~d}$ & $1,98 \mathrm{c}$ \\
\hline \multicolumn{3}{|l|}{ A n Flise de Vari ncia (resumo) } \\
\hline$F($ tratam entos) & $286,00 * *$ & 346,69 ** \\
\hline D M S & 1,08 & 1,14 \\
\hline$C \vee(\%)$ & 30,69 & 27,83 \\
\hline
\end{tabular}

* Dados de porcentagem transformados em $\sqrt{x+0,5} . \quad$ ** Significativo ao nível de $5 \%$ de probabilidade.

DMS = Diferença mínima significativa. $\quad$ CV $=$ Coeficiente de variação. 
Ao contrário do observado para ambas as doses do oxassulfurom na leitura realizada 20h após incubação, o glifosato não afetou a porcentagem de germinação dos conídios de $M$. anisopliae na segunda leitura (48 h), embora tenha reduzido de forma expressiva sua viabilidade. O mesmo fato ocorreu com o oxassulfurom a $30 \mathrm{e} 60 \mathrm{~g} / \mathrm{ha}$.

Apesar de serem incompatíveis com o crescimento e a reprodução de $M$. anisopliae, glifosato e oxassulfurom não afetaram a viabilidade de seus conídios. A incongruência entre esses parâmetros deve-se ao fato de que somente o crescimento vegetativo e a esporulação do fungo são incluídos na fórmula para o cálculo do fator T. De acordo com BATISTA FILHO, LAMEIDA e LAMAS (2003), outros parâmetros como a viabilidade e a virulência do entomopatógeno deveriam ser considerados no estabelecimento da compatibilidade.

\title{
4 CONCLUSÃO
}

Os herbicidas butafenacil, 2,4-D, metribuzim, s-metolacloro e trifloxissulfurom de sódio reduziriam significativamente tanto o crescimento vegetativo como a reprodução do fungo, mostrandose inviáveis para a sobrevivência do $M$. anisopliae.

Os herbicidas glifosato na dose de $0,5 \mathrm{~L} / \mathrm{ha}$ e oxassulfurom a 30 e $60 \mathrm{~g} / \mathrm{ha}$ não afetaram o crescimento vegetativo de $M$. anisopliae.

Todos os tratamentos foram classificados como tóxicos ou muito tóxicos ao fungo, apesar do glifosato e do oxassulfurom não afetarem a viabilidade dos conídios.

\begin{abstract}
EFFECT OF HERBICIDES UTILIZED IN SUGAR CANE OF "IN VITRO" DEVELOPMENT OF ENTOMOPHATOGENIC FUNGI Metarhizium anisopliae (Metsch.) SOROKIN

The compatibility of the herbicides butafenacil, metribuzin, s-metolachlor, 2,4-D, glyphosate, oxasulfuron and trifloxysulfuron in the development of entomopathogenic fungi $M$. anisopliae (isolate IBCB 348) were evaluated, which is employed in the biological control of Mahanarva fimbriolata (Hemiptera: Cercopidae). The herbicides were added to the culture media potato dextrose agar (PDA) in temperature of approximately $45^{\circ} \mathrm{C}$ in minimal and maximal recomended field doses, fixing the volume of $200 \mathrm{~L} / \mathrm{ha}$. After the solidification of the culture media in Petri dishes, the fungi was inoculated in three equidistant points. The plates were incubated in climatized chamber and the average diameter of the formed colonies were evaluated, the number of conidia per colony and the viability of the conidia after 20 and 48 hours inoculation. Only the herbicide oxasulfuron, in the lowest tested dose $(30 \mathrm{~g} / \mathrm{ha})$ was classified as toxic to $M$. anisopliae, being the rest classified in both doses as highly toxic. For viability only the herbicides oxasulfuron in the doses of 30 and $60 \mathrm{~g} / \mathrm{ha}$ didn't affect the germination of the fungi until 20 hours after inoculation and glyphosate at 0.5 and $6.0 \mathrm{~L} / \mathrm{ha}$ until 48 hours. The other herbicides were imcompatible, affecting the development and the germination of $M$. anisopliae.
\end{abstract}

KEY-WORDS: HERBICIDES; SUGAR CANE; Metarhizium anisopliae (Metsch.) SOROKIN.

\section{REFERÊNCIAS}

1 ALVES, R.T.; BATEMAN, R.P.; PRIOR, C.; LEATHER, S.R. Effects of simulated solar radiation on conidial germination of Metarhizium anisopliae in different formulations. Crop Protection, v. 17, n. 8, p. 675-679, 1998.

2 ALVES, S.B.; MOINO JR., A.; ALMEIDA, J.E.M. Produtos fitossanitários e entomopatógenos. In: ALVES, S.B. (Ed.). Controle microbiano de insetos. Piracicaba: FEALQ, 1998. Cap.8, p.217-238.

3 ÁVILA, J. C.; MELHORANÇA, A. L. Eficiência do vírus de poliedrose nuclear em mistura com herbicidas pósemergentes no controle de Anticarsia gemmatalis Hübner (Lepidópetra: Noctuidae). An. Soc. Entomol. Brasil, v. 28, n. 2, p. 339-341, 1999.

4 BANZATTO, D.A.; KRONKA, S.N. Experimentação agrícola. Jaboticabal: FUNEP, 1989. 247 p.

5 BATISTA FILHO, A.; ALMEIDA, J.E.M.; LAMAS, C. Effect of thiamethoxam on entomopathogenic microorganisms. Neotropical Entomology, v. 30, n. 3, p. 437-447, 2001.

6 COSTA, E.A.D.; ALMEIDA, J.E.M.; LOUREIRO, E.S.; SANO, A.H. Avaliação da compatibilidade dos adjuvantes AG 6202 E AGRHOTM DEP-775 no desenvolvimento "in vitro" dos fungos entomopatogênicos Metarhizium anisopliae (Metsch.) Sorokin e Beauveria bassiana (Bals.) Vuillemin. In: CONGRESSO NACIONAL DA SOCIEDADE DOS TÉCNICOS AÇUCAREIROS E ALCOOLEIROS DO BRASIL, 8., Recife. Anais... Recife: STAB Setentrional, 2002. p. 67-72.

7 MACEDO, N.; CAMPOS, M.B.S.; ARAÚJO, J.R. Insetos nas raízes e colo da planta, perfilhamento e produtividade em canaviais colhidos com e sem queima. STAB Açúcar, Álcool e Subprodutos, v. 15, n. 3, p. 18-21, 1997.

8 MATALLO, M. B. La relación suelo - herbicida: descoposición, resíduos, contaminación y manejo. In: CONGRESO DE LA ASSOCIACION LATINOAMERICANA DE MALEZAS, 15., 1999, Cartagena de Indias, Colombia. Memórias... Cartagena de Índias: [s.n.], 1999. p. 3-8. 